\title{
LS Channel Estimation in Multicell Multiuser MIMO OFDM with Zadoff Chu Sequence as Pilots
}

\author{
Saarika B S ${ }^{1}$, Anu Anna John ${ }^{2}$ \\ Department of Electronics and Communication Engineering,MarBaselios College of Engineering and \\ Technology, Thiruvananthapuram, India
}

\begin{abstract}
High spectral efficiency and the ability to combat multipath fading are the key requirements of future wireless communication systems. Multiple-input multiple-output (MIMO) combined with orthogonal frequencydivision multiplexing (OFDM) has been widely considered to be a promising candidate. Moreover Multi user MIMO OFDM can provide good spatial multiplexing with single antenna for each user. In the case of multicell multiuser MIMO OFDM system main challenge is to acquire the channel state information. In order to get the channel state information, channel estimation is to be done. Here pilot aided LS channel estimation is used to obtain the estimates. For multi cell multi user MIMO OFDM, channel estimation using pilot subcarriers causes pilot contamination. This is due to the reuse of pilot subcarriers in different cells. In order to reduce the impact of pilot contamination, pilot sequences must select properly. Here Zadoff Chu sequences are used for the design of pilots in different cells.
\end{abstract}

Keywords: Channel estimation, LS channel estimation, MIMO, OFDM, Pilot contamination

\section{Introduction}

Requirements of the future wireless communication system are to provide a reliable communication along with high data rate transmission. As MIMO along with OFDM can provide good data rate transmission and also it has the ability to attain good spectral efficiency, it is considered as good solution [1]. As multiuser MIMO has got more resistance to wireless propagation conditions and also minimal cost at the terminals than point to point MIMO, it is used in future wireless communication systems. Channel state information is necessary for obtaining good performance in any communication system, likewise in multicell scenario also [2]. Allocation of dedicated pilots for the users in each cell is difficult, due to the mobility of users and bandwidth limitation. Thus pilots are reused among users of different cells.

The reuse of pilot causes pilot contamination (PC), which is caused by the assignment of nonorthogonal pilots for users in different cells. With these non-orthogonal pilots, the channel estimate at the base station of one cell got contaminated by the pilots of the users in other cells [3]. Pilot contamination occurs only in multicell scenario and it is lost if the area of consideration is a single cell. The performance of the system is severely affected by the pilot contamination than that due to channel noise. By increasing the number of base station antennas will remove the effects of fast fading and uncorrelated interference but pilot contamination due to the use non orthogonal pilots in other cells won't vanish. The perfect knowledge of channel state information at the base station is a necessity, as it can improve reliability and power efficiency can be improved [4]-[6].

In (7), in order to reduce the effects of inter cell interference in MIMO OFDM pilot based on chu sequences are employed. These Chu sequence has a property that it has zero autocorrelation with its cyclic shifted ones. Here in this work, uplink transmission of multicell multiuser MIMO OFDM along with channel estimation, pilot contamination, multicarrier and multipath is considered. First, for estimating the channel known sequences are inserted into the information bits known as pilot sequences. These pilot sequences are inserted into the information bits of each users. In a multicell scenario with $\mathrm{L}$ cells and $\mathrm{K}$ users in each cell, number of orthogonal pilots required will be $\mathrm{K} \times \mathrm{L}$. As it is not possible, non- orthogonal pilots are being used for users in different cells. Then LS channel estimation is done for obtaining the channel estimates at the base station. As pilots are reused the estimates obtained at the base station of one cell get contaminated by the users of the other cells. Thus pilot contamination occurs. Here in order to reduce the impact of pilot contamination in LS channel estimation Zadoff Chu sequences are used as pilot sequences for users in different cells avoiding the reuse of pilot sequences. This is possible because of the properties associated with the Zadoff Chu sequences. After using Zadoff Chu sequence as pilot sequence, LS channel estimation is done and the mean square error of the channel estimate is obtained. from the results obtained it is clear that the impact of pilot contamination is reduced. 


\section{System Model}

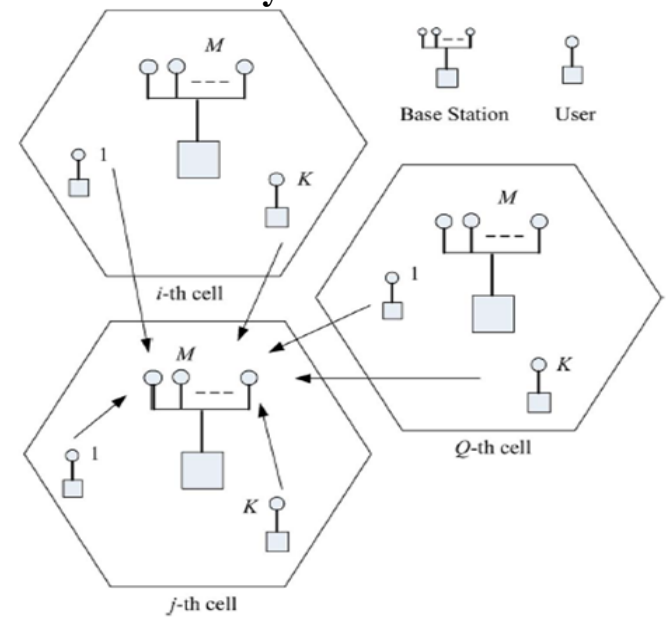

Fig.1 Multicell Multiuser MIMO Uplink Transmission

Consider a multicell multiuser MIMO OFDM system with Q cells as shown in fig 1 . In each cell there is a base station with $\mathrm{M}$ antennas and $\mathrm{K}$ users with single antenna terminals. Also with the number of antennas at the base station $\mathrm{M}$ greater than or equal to the number of single antenna terminal users $\mathrm{K}$ at each cell. $\mathrm{N}$ subcarrier OFDM is considered. The channel is modeled as a frequency selective channel with finite channel impulse response with $\mathrm{L}$ taps. We consider an uplink transmission in which $\mathrm{q}^{\text {th }}$ base station receives signal from the users of all cells.

The received signal vector of size $N \times 1$ on all $N$ subcarriers at the $r^{\text {th }}$ antenna at the $j^{\text {th }}$ BS can be expressed as; $Y_{j}=X H_{j}+Z_{j}(1)$

where $Y_{j}=\left[Y_{j}(0), \ldots, Y_{j}(N-1)\right]^{T}, X=\left[X_{1}, \ldots, X_{Q}\right], X_{q}$ is a diagonal matrix.it contains the transmit signal from the $q^{\text {th }}$ cell and $Z_{j}=\left[Z_{j}(0), \ldots . Z_{j}(N-1)\right]^{T}$ is a vector of independently and identically distributed (i.i.d.) complex zero-mean Gaussian noise variables with variance $\sigma^{2} \cdot H_{j}=\left[H_{j 1}{ }^{T}, \ldots, H_{j Q}{ }^{T}\right]^{T}, H_{j q}$ is the frequency response of the channel between the $j^{t h}$ and $q^{t h}$ cells. $H_{j q}=\left[H_{j q 1}{ }^{T}, \ldots \ldots, H_{j q k}{ }^{T}\right]^{T}$

The received signal at the $\mathrm{j}^{\text {th }} \mathrm{BS}$ can be rewritten as;

$$
Y_{j}=\sum_{q=1}^{Q} \sum_{k=1}^{K} X_{q k} H_{j q k}+Z_{j}
$$

Let $X_{q k}=S_{q k}+B_{q k}$ where $S_{q k}$ is an arbitrary $N \times N$ data diagonal matrix, and $B_{q k}$ is an $N \times N$ pilot diagonalmatrix. where $T_{q}=\left[S_{q 1} F_{N, L}, \ldots, S_{q K} F_{N, L}\right], A_{q}=\left[B_{q 1} F_{N, L}, \ldots, B_{q K} F_{N, L}\right]$

\section{LS ESTIMATION}

Here we are taking the assumption that all subcarrier has the same power and for different users in each cell, phase shifted orthogonal pilot sequences are used and these same pilot sequences are reused for users in other cells;

$A_{j}{ }^{t} A_{q}=I_{L K}\left(A^{t}=\left(A^{H} A\right)^{-1} A^{H}\right)$ and $A_{j}{ }^{t} T_{q}=0_{L K}(3)$

where $A_{q}=\left[B_{q 1} F_{N, L}, \ldots, B_{q K} F_{N, L}\right]$

By multiplying $A_{j}{ }^{\dagger}$ in both sides of Eq (3), channel vector between $j^{\text {th }}$ BS and K users in the $j^{\text {th }}$ cell is obtained as;

$$
\hat{C}_{j j}{ }^{L S}=A_{j}{ }^{t} Y_{j}(4)
$$

The MSE of the LS channel estimates is obtained by taking the mean of the squared error between the estimated channel coefficients and the actual coefficients. As pilots are reused, pilot contamination occurs. Appropriate pilot design method or pilot allocation schemes can be used to reduce the impact of pilot contamination in LS channel estimation.

\section{Pilot Design Using Zadoff Chu Sequence To Reduce Pilot Contamination}

Zadoff Chu sequences can be used as pilot tones in multicell multiuser MIMO due to its correlation properties. If the pilot length is not less than the number of users $\mathrm{K}$ in each cell, then the pilot tones for users in a cell will be orthogonal due to the cyclic shifts applied and these pilots are reused for users in others cells after applying phase shifts. 
First a Chu sequence of length $\mathrm{N}_{\mathrm{p}}$ is generated as $\mathrm{z}=\left[z_{0}, z_{1}, \ldots z_{N_{p}-1}\right]$.The $\mathrm{n}^{\text {th }}$ entry of the Chu sequence is represented by;

$z_{n}=\exp \frac{i N \pi}{N_{p}} n\left(n+\left(N_{p} \bmod 2\right)\right), n=0,1, \ldots . N_{p}-1$

Where $N_{p}$ represents the length of the sequence and $\mathrm{N}$ represents the unique sequence parameter. This $\mathrm{N}$ should be relatively prime with the length of the sequence. The circular autocorrelation of the sequence $\mathrm{z}$ is defined as; $r_{j}=\sum_{n=0}^{N_{p}-1} z_{n} z_{(n+j)}{ }^{*} \bmod N_{p}, j=0,1, \ldots . N_{p}-1$

For value of $\mathrm{j}=0$ only the autocorrelation value is $N_{p}$ and for all other values of $\mathrm{j}$, autocorrelation values $r_{j}=0$. A new sequence $\mathrm{z} 1=\left[z 1_{0}, z 1_{1}, \ldots \ldots . z 1_{N_{p}-1}\right]$ is formed with $z_{n}$ as;

$z 1_{n}=z_{n} \exp \frac{i 2 \pi l n}{N_{p}}, n=0,1, \ldots . N_{p}-1$

Thus from a root sequence, new sequences can be formed by applying different phase shifts for different cells. The new sequences along with its cyclic shifted versions can be used as pilot sequences for users in other cells.

Let the pilot vector for one user in a cell and the $\mathrm{n}^{\text {th }}$ entry can be denoted as;

$\left[P_{l}\right]_{n}=z_{n} \exp \frac{i 2 \pi l_{q} n}{N_{p}}, n=1,2, \ldots . . N_{p}-1(8)$

Where $\exp \frac{i 2 \pi l_{q} n}{N_{p}}$ represents the phase shift. qiis an integer which is not divisible by $N_{p}$ and differs for each cell $\mathrm{q}, \mathrm{q}=1,2, \ldots \mathrm{Q}$. Then the designed pilots are cyclically shifted for users inside the cell. For different cells the value of $l_{q}$ is different and thus the phase shifts will be different.

\section{Experimental Results}

In order to find the effect of pilot contamination in the MSE of the channel estimates, multicell multiuser MIMO is considered with $\mathrm{M}$ antennas at the base station. Here, number of cells $\mathrm{Q}$ is taken as 2 and each cell consists of two users with single antenna terminals. Also the base station at each cell consists of three antennas. OFDM symbol with $\mathrm{N}=128$ is considered and the cyclic prefix length is taken as 16 . Length of pilot sequence is taken as 8 and the pilot type is comb.

\subsection{Mse Performance}

The MSE performance of LS channel estimation versus SNR is plotted for different values of channel length $\mathrm{L}$ is shown in fig. 2. Number of OFDM subcarriers $\mathrm{N}$ is taken as 128 and value of $\mathrm{L}$ is taken as 4,8 and 16. The performance of LS channel estimates is improved when the channel length is increased.Orthogonal phase shifted pilot sequences are used for users in a cell and the same pilots are reused among the users of different cells. As pilot sequences are reused, pilot contamination occurs. The effect of pilot contamination got reduced by increasing the channel length.

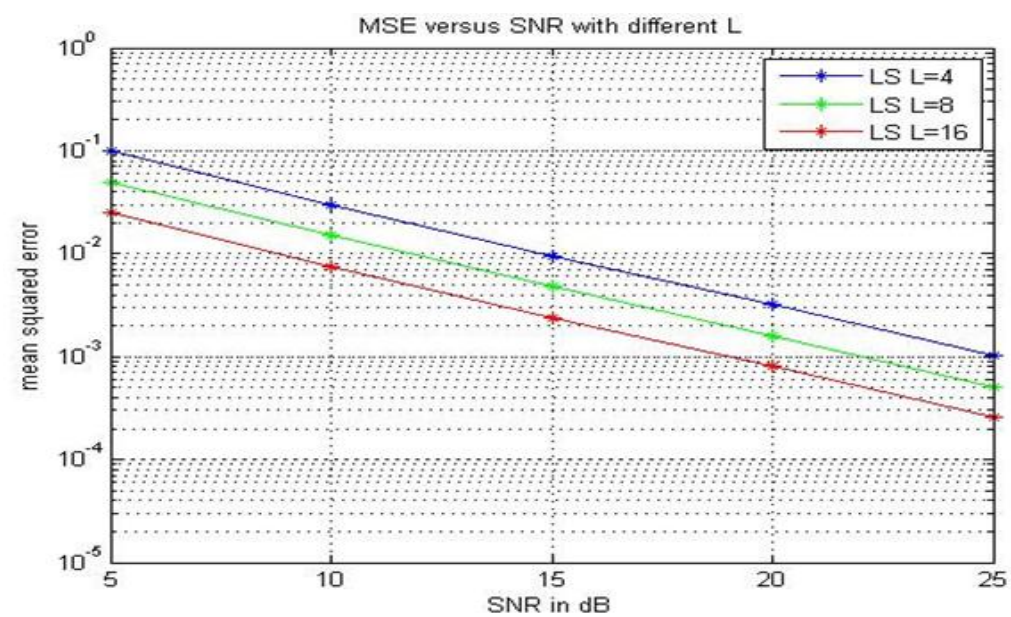

Fig. 2 MSE Versus SNR performance of LS channel estimation

The MSE performance of LS channel estimates versus SNR is plotted after using Zadoff Chu sequence as pilot tones. As due to the properties of the Zadoff Chu sequence orthogonal pilot sequences are assigned to users in different also. Thus the impact of pilot contamination is reduced. It is evident that MSE performance get improved with the use of Chu sequence as pilot tones. A performance improvement of $6 \mathrm{~dB}$ is obtained. 


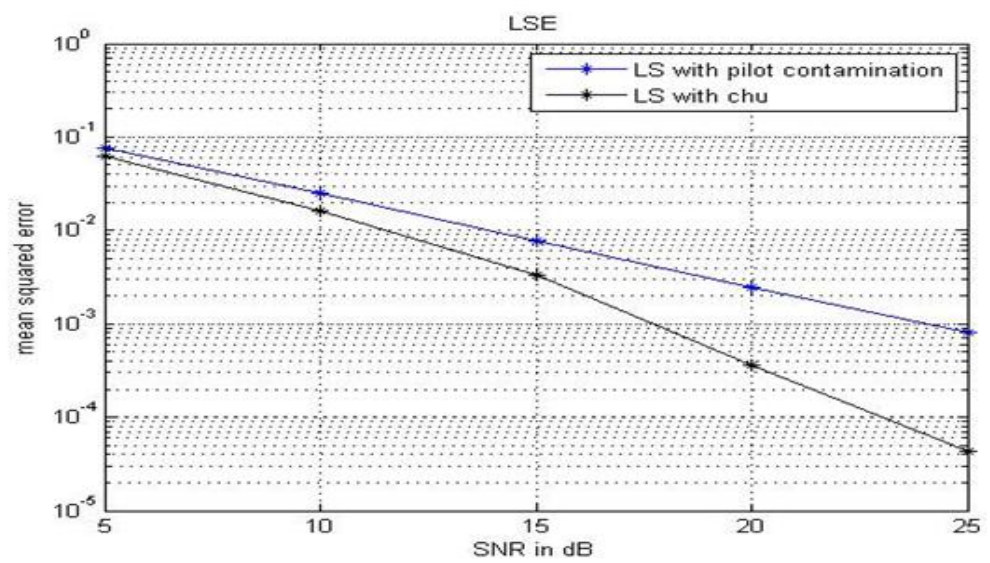

Fig.3 MSE versus SNR plot of LS channel estimation with Chu

\section{Conclusion}

In this paper, channel estimation in multicell multiuser MIMO OFDM is studied. In multicell multiuser MIMO OFDM system, pilot contamination occurs due to the reuse of pilot in different cells. Therefore the impact of pilot contamination on the pilot based LS channel estimation algorithms is considered. From the experimental results it can be seen thatpilot contamination in LS channel estimation can be significantly reduced by changing the channel lengths. Here a channel estimation technique with a new design for pilot is proposed which can suppress pilot contamination in multicell multiuser MIMO OFDM scenario. Chu sequence is used as pilot based on the property that it exhibit zero auto correlation with it cyclic shifted versions. Hence orthogonal pilots can be employed for users in different cells and thus pilot contamination can be significantly reduced.

\section{References}

[1] D. Gesbert, M. Shafi, D. Shiu, and P. J. Smith, "From theory to practice: An overview of MIMO space-time coded wireless systems," IEEE J. Sel.AreasCommun., vol. 21, no. 3, pp. 281-302, Apr. 2003

[2] D. Gesbert, M. Kountouris, R. W. Heath, Jr., C. B. Chae, and T. Sälzer, "From single user to multiuser communications: Shifting the MIMO paradigm," IEEE Signal Process. Mag., vol. 24, no. 5, pp. 36-46,Oct. 2007.

[3] PengXu, Jiangzhou Wang, Jinkuan Wang, "Analysis and Design of Channel Estimation in Multicell MU-MIMO OFDM Systems", IEEE Transactions on Vehicular Technology, Vol. 64, No. 2, February 2015.

[4] J. Jose, A. Ashikhmin, T. L. Marzetta, and S. Vishwanath, "Pilot contamination and precoding in multi-cell TDD systems," IEEE Trans. WirelessCommun., vol. 10, no. 8, pp. 2640-2651, Aug. 2011.

[5] H. Yin, D. Gesbert, M. Filippou, and Y. Liu, "A coordinated approach tochannel estimation in large-scale multiple-antenna systems," IEEE J. Sel.AreasCommun., vol. 31, no. 2, pp. 264-273, Feb. 2013.

[6] H. Ngo, T. L. Marzetta, and E. G. Larsson, "Analysis of the pilot contamination effect in very large multicell multiuser MIMO systems for physical channel models," in Proc. ICASSP, Prague, Czech Republic,May 2011, pp. 3464-3467.

[7] G. Kang, P. Hasselbach, Y. Yang, P. Zhang, and A. Klein, "Pilot design for inter-cell interference mitigation in MIMO OFDM systems," IEEECommun. Lett., vol. 11, pp. 237-239, Mar. 2007.

[8] W. Wang, X. Wu, and G. Kang, "Pilot sequence design scheme for inter-cell interference mitigation in OFDM systems under timevarying channels," in Proc. IEEE Vehicular Technology Conf. (VTC 2008),Singapore, 2008, pp. 314-318. 